\title{
Discussion: Pedestrian-induced vibrations of the Clifton Suspension Bridge, UK
}

\author{
J. H. G. Macdonald \\ Contribution by A. N. Beal, Thomasons
}

One significant difference between the Clifton Suspension Bridge and the London Millennium Bridge is that the former has cables and hangers which are hung vertically, whereas the latter is suspended from angled cables. For a bridge with vertical cables, the primary movement induced by a vertical load is up and down only, but with angled cables, an off-centre vertical load will also cause lateral movements, which are much more disturbing for pedestrians. Did the author consider this type of effect in his analysis?

\section{AUTHOR'S REPLY}

The coupling of torsional and lateral motion was only considered in the paper in defining the mode shapes of the Clifton Suspension Bridge (CSB), as given in Figure 5 of the paper. On virtually all bridges there is some coupling of torsional and lateral motion in combined modes, although usually each mode is dominated by one type of motion with only a minor component of the other, as found on the CSB.

On the London Millennium Bridge (LMB) the torsional effect described by the discusser is less significant than suggested since the deck cross-section is rigidly attached to the cables by outriggers able to carry bending moments, rather than suspended on hangers that act as pin-ended. Furthermore the majority of the bridge's stiffness is from the geometric stiffness of the cables ${ }^{2,10}$ and since the cables are under high tension, they have similar transverse stiffness in the inclined cable plane and perpendicular to it. Hence torsional and lateral motions are not so directly coupled in a mechanism as suggested.

Nevertheless, it is reported that some coupling of lateral and torsional motion did occur in some modes, but this and the response to eccentrically applied vertical loads were considered by Arup at the design stage. ${ }^{2}$ The measured dynamic properties of the bridge were subsequently found to be very similar to those predicted. The forcing due to torsion was estimated to be small relative to the direct lateral excitation. ${ }^{10}$

Regarding the pedestrian response to the motion, in laboratory tests McRobie et al. ${ }^{11}$ found little difference in the behaviour of pedestrians walking on a deck section model suspended by inclined as opposed to vertical pin-ended hangers. They concluded that the rotational part of the motion was not very significant.
It is now clear that the lateral excitation mechanism is independent of the structural form of the bridge. Since the well publicised LMB episode, numerous other examples of the phenomenon have come to light on bridges of different structural forms, including arch, truss, box girder, cable-stayed and suspension bridges (see, for example, the cases cited in Dallard et al. $^{2,10}$ and Blekherman ${ }^{12}$ ).

The pedestrian model proposed by Barker, ${ }^{9}$ which does not require synchronisation, has now been enhanced by considering the mechanism of human balance, drawing on findings from biomechanics research. The resulting pedestrian model $^{13}$ gives additional forces on the bridge equivalent to negative damping, in agreement with Arup's empirical model from back-calculation of the LMB measurements (and as also found on the CSB by the author). This model is based entirely on the lateral component of bridge motion, which affects the lateral balance of the pedestrian, leading to the additional lateral forces. A vertical component of bridge motion would have negligible effect on pedestrian balance. For a coupled lateral/torsional mode, the torsional component would add to the modal mass, increasing the critical number of pedestrians required for dynamic instability. Eccentric vertical forcing would increase the background level of vibrations but it would not affect the dynamic instability, which is the cause of the large amplitude vibrations. The instability occurs when the total damping becomes negative, the negative damping from the pedestrian being associated with the lateral component of motion but being independent of the background amplitude.

\section{REFERENCES}

2. Dallard P., Fitzpatrick A. J., Flint A., Le Bourva S., Low A., RIDSDill SMith R. M. and Willford M. The London Millennium Footbridge. The Structural Engineer, 2001, 79, No. 22, 17-33.

9. BARKER C. Some observations on the nature of the mechanism that drives the self-excited lateral response of footbridges. Proceedings of the 1st International Conference on Design \& Dynamic Behaviour of Footbridges, Paris, November 2002.

10. Dallard P., FitzPatrick A. J., Flint A., Low A. and Ridsdill Sмгтн R. M. The Millennium bridge, London: problems and 
solutions. The Structural Engineer, 2001, 79, No. 8, 15-17.

11. McRobie A., Morgenthal G., Lasenby J. and Ringer M. Section model tests on human-structure lock-in.

Proceedingsof the Institution of Civil Engineers, Bridge Engineering, 2003, 156, No. BE2, 71-79.
12. BLEKHERMAN A. N. Swaying of pedestrian bridges. ASCE Journal of Bridge Engineering, 2005, 10, 142-150.

13. Macdonald J. H. G. Lateral excitation of bridges by balancing pedestrians. Proceedings of the Royal Society A, 2009, 465, 1055-1073. 